\title{
Comparison Between Automated and Manual Sphygmomanometer for Measuring Blood Pressure in Children
}

\author{
Taksande A $^{1}$, Jadhav A $^{2}$, Vagha J ${ }^{3}$
}

\begin{abstract}
Introduction: The mercury sphygmomanometer has been the gold standard used for obtaining blood pressure (BP). However, due to environmental concerns and more use of automated $\mathrm{BP}$ devices, an alternative to using the standard mercury sphygmomanometer to measure BP. The aim of this study is to evaluate the accuracy of the Automated (Omron) BP device against the mercury sphygmomanometer in children. Material and Methods: One hundred children were enrolled in this study. The accuracy of Omron HEM-7121 BP readings was compared with that of mercury BP device readings. Each children had four BP measurements recorded sequentially i.e. two valid systolic readings, and two valid diastolic readings. Results: BP taken with the automated device was systolic $109.77 \pm 9.97$ and diastolic $74.50 \pm 8.32 \mathrm{~mm} \mathrm{Hg}$ compared to systolic $112.68 \pm 9.98$ and diastolic $77.38 \pm 7.91 \mathrm{~mm} \mathrm{Hg}$ measured by manual mercury sphygmomanometer BP readings $(p<0.001)$. Automated and mercury BP measurements were correlated ( $r=0.89$, systolic $\mathrm{BP} ; r=0.82$, diastolic $\mathrm{BP}$ ). Linear regression analysis showed that the automated systolic BP is a significant predictor $(\beta=0.897$, $p<0.001)$ of manual systolic BP. Similarly, automated diastolic BP was also a significant predictor $(\beta=0.829, p<0.001)$ of manual diastolic BP. Conclusion: This study concludes that the automated BP device is reliable and accurate for measuring the $\mathrm{BP}$ in children.
\end{abstract}

Key words: Automated devices; blood pressure; measurement; OMRON, Mercury Sphygmomanometer

\section{Introduction}

Blood pressure (BP) is seen as one of the vital signs of life. An accurate assessment of BP is very important for the diagnosis and treatment of hypertension. BP measurement can be taken by both invasively and non-invasively, but it requires careful attention and dependent on proper use of the equipment. Hypertension is the most common risk factor for cardiovascular disease, accurate BP measurement is essential in providing healthcare to decrease the risks of cardiovascular morbidity and mortality ${ }^{1}$. The importance of attaining the diagnosis of high BP and its continuous monitoring are well known. Therefore, several equipments have been available for the measurement of BP, not only to be used by specialized
'Dr. Amar Taksande, MD, FIAE, Fellow in Paediatric Cardiology, Professor, ${ }^{2}$ Dr. Aishwarya Jadhav, MBBS, Senior Resident, ${ }^{3}$ Dr. Jayant Vagha, Professor and Head of department. All from the department of Paediatrics, Jawaharlal Nehru Medical College, Sawangi Meghe, Wardha, Maharashtra, India.

\author{
Address for correspondence: \\ Dr. Amar M Taksande, Professor \\ Department of Paediatrics \\ Jawaharlal Nehru Medical College, \\ Sawangi Meghe, Wardha, \\ Maharashtra - 442102, India \\ E mail: amar.taksande@gmail.com \\ Tel No: +917152-27705
}

\section{How to cite}

Taksande A, Jadhav A, Vagha J. Comparison Between Automated and Manual

Sphygmomanometer for Measuring Blood Pressure in Children. J Nepal Paediatr Soc 2015;35(1):13-17.

doi: http://dx.doi.org/10.3126/jnps.v35i1.11568

This work is licensed under a Creative Commons Attribution 3.0 License.

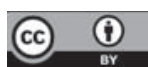

individuals, but also by the population itself ${ }^{2}$. Although digital monitors are easier to use when compared to the mercury sphygmomanometers, it is essential to establish their reliability and validity. Traditionally, BP have been measured using a manual mercury sphygmomanometer, but in recent years the use of automated BP machines such as the Dinamap and Omron have been the trend in most hospitals ${ }^{3}$. However, many health workers question the accuracy and reliability of the automated machines when used in the clinical setting, 
and still prefer the manual sphygmomanometer which is considered the 'gold standard'when used by a trained observer ${ }^{4,5,6}$. There is some evidence in the literature that digital monitors are reliable and accurate when compared with other devices, such as the aneroid or mercury devices ${ }^{6,7,8,9}$. This type of equipment can replace the manual sphygmomanometer in some contexts, such as at home or in epidemiological studies within the community. However, most studies were done only on adults, and therefore, the reliability of automated blood pressure machines among children's is unclear. This study was done to compare the blood pressure measurement measured by an Automated and Manual Sphygmomanometer in children and to determine the reliability of automatic BP machine for measuring the BP.

\section{Material and Methods}

This cross-sectional study study was conducted in the paediatric department at Acharya Vinoba Bhave Rural Hospital which is a rural medical college located in Sawing, Maharashtra from September 2013 to March 2014. Inclusion criteria: Children were age 3-15 years and no known history of serious illness. Exclusion criteria: Upper limb amputation, cuts or bruising of the skin at measurement sites, arrhythmia, aortic coarctation, aortic dissection, peripheral vascular disease, congenital heart disease and vasculitis.

BP measurements were taken by postgraduate student in paediatrics using:-

1. The manual device which was used is a standard mercury sphygmomanometer (M) with an appropriate cuff and Littman Classic II stethoscope.

2. The automated device (A) which was used is the OMRON machine with different size of cuffs [HEM-CS24 17-22cm (7-9inch) \& HEM-CR24 22-32 $\mathrm{cm}$ (9-13inch)]

3. All machines which were used in the study were calibrated and checked for compliance to machine standards by the Biomedical Engineering Department of the Hospital.

Measurement of Blood Pressure: Children were positioned supine, with arms straight and legs uncrossed. Appropriate size of the cuff was selected by measuring mid arm circumference at the halfway point between shoulder tip and elbow tip. After that, we fitted the cuff to the patient's arm.

a. By Mercury sphygmomanometer (M): Firstly, we determined a 'systolic estimate' of the children BP by inflating the cuff until the radial pulse can no longer be palpated. Then, again inflate the cuff a further $30 \mathrm{mmHg}$ and release the valve at $2 \mathrm{mmHg}$ per second until the radial pulse reappears. We recorded this reading as the systolic BP estimate.

b. Again we inflated the cuff to $30 \mathrm{mmHg}$ higher than the 'systolic estimate' and then slowly deflated the cuff at $2 \mathrm{mmHg}$ per second and, using a stethoscope, record measures corresponding to the $1^{\text {st }}$ and 5 th Korotkoff sounds. $1^{\text {st }}$ Korotkoff sound was considered as systolic BP and $5^{\text {th }}$ Korotkoff sound was considered as diastolic BP. If Korotkoff sounds persist, the measurement was repeated with less pressure on the stethoscope head. If the sounds persist at low intensity, then K4 (muffling of sounds) was recorded as the diastolic pressure.

c. By Automatic blood pressure machine (A): The Omron HEM-7121 automatic measurements are based on smart "inflate" technology (IntelliSense), where inflation is driven by a pumping system and deflation is driven by an electromagnetic control valve that allows rapid air release. We pressed 'start' button on machine to commence BP measurement. Record was digitally displayed as systolic and diastolic BP.

Frequency of BP Measurement: For each person, the average of the two measurements were calculated from the two devices, which was leads to four readings per subject denoted as systolic manual, systolic automated, diastolic manual, and diastolic automated. Half of the BP was measured first with the digital and subsequently with the mercury one, whereas the remaining half was evaluated in the opposite order. Heart rate was also obtained. There was a two minutes break between automatic and manual BP readings.

To detect a mean difference between measurement techniques of $5 \mathrm{mmHg}(10 \%)$ at a two tailed alpha of 0.05 , power of $80 \%$ the estimated sample size of 96 was required.

Statistical Analysis: SPSS-14 software was used for the statistical analysis. Means and standard deviations of manual and automated systolic and diastolic BP values were calculated. A paired $t$-test was used to assess the differences between the manual BP and automated BP readings. Pearson's correlation coefficient was used to determine the relationship between BP's measured by manual and automated BP device. Bland-Altman plots were used to show the distribution of the differences between the methods at all pressures and the mean and standard deviation of the differences. A linear regression analysis was performed to examine the 
relationship between the automated and manual BP readings with the automated systolic and diastolic BPs as the independent variables.

The research was approved by an Institutional Ethics Committee (IEC) and informed consent was obtained from the parents.

\section{Results}

Out of the 100 children studied, 48 were boys and 52 were girls. The age distribution ranged from 5-15 years. Mean age of boys and girls were $10.04 \pm$ 2.94 and $9.85 \pm 3.04$ years, respectively. Mean weight was $27.16 \pm 8.96$, mean height was $129.60 \pm 17.09$ $\mathrm{cm}$ and mean circumference of arm was $18.01 \pm 1.72$ $\mathrm{cm}$ (range 15-22 cm). BP taken with the automated OMRAN device was systolic $109.77 \pm 9.97$ and diastolic $74.50 \pm 8.32 \mathrm{~mm}$ Hg compared to systolic $112.68 \pm 9.98$ and diastolic $77.38 \pm 7.91 \mathrm{~mm} \mathrm{Hg}$ measured by manual mercury sphygmomanometer BP readings $(p<0.001)$. The comparison of the manual versus the automated readings showed a high coefficient of correlation $(r 2)$ for systolic BP $(r 2=0.80)$ and diastolic BP $(r 2=0.76)$ readings.

Bland-Altman plot (Fig. 1 and 2) demonstrates the mean difference between systolic and diastolic blood pressure measured by Mercury Sphygmomanometer and automated BP device (OMRON). Black line is the mean of the differences; dotted lines are both lower and upper $95 \%$ confidence interval. If the differences within mean \pm 1.96 SD are not clinically important, the two methods may be used interchangeably.

Linear regression analysis (Fig. 3) showed that the automated systolic BP is a significant predictor $(\beta=$ $0.897, p<0.001$ ) of manual systolic BP with a regression equation (systolic BP $=14.11+0.89 \times$ automated systolic). Also, Automated diastolic BP was also a significant predictor $(\beta=0.829, p<0.001)$ of manual diastolic BP with a regression equation (Diastolic BP = $15.59+0.82 \times$ automated diastolic).

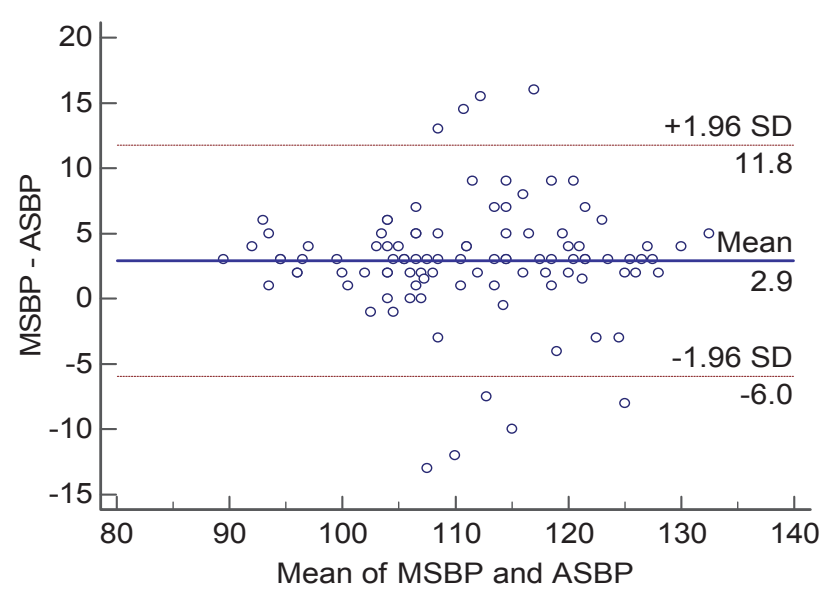

Fig 1: Bland and Altman plot showing difference of systolic BP (SBP) between mercury sphygmomanometer and automated device.

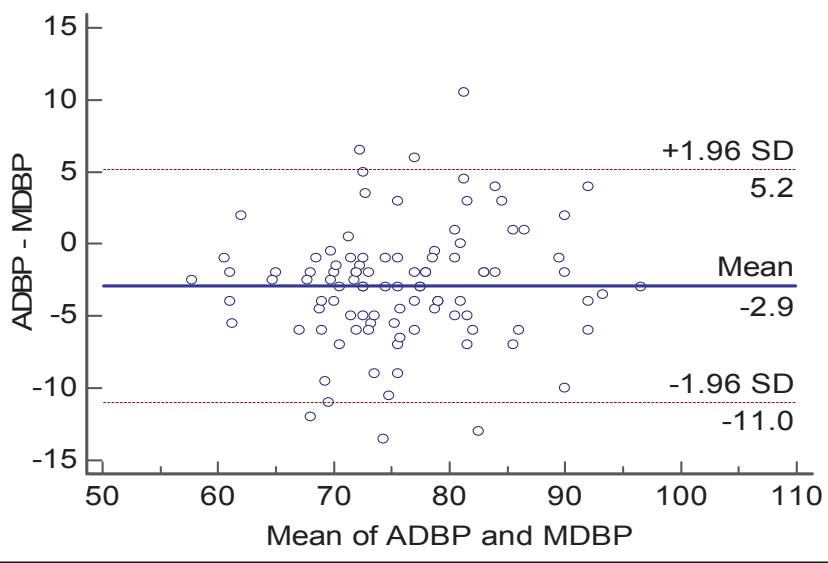

Fig 2: Bland and Altman plot showing difference of diastolic BP (DBP) between mercury sphygmomanometer and automated device. 


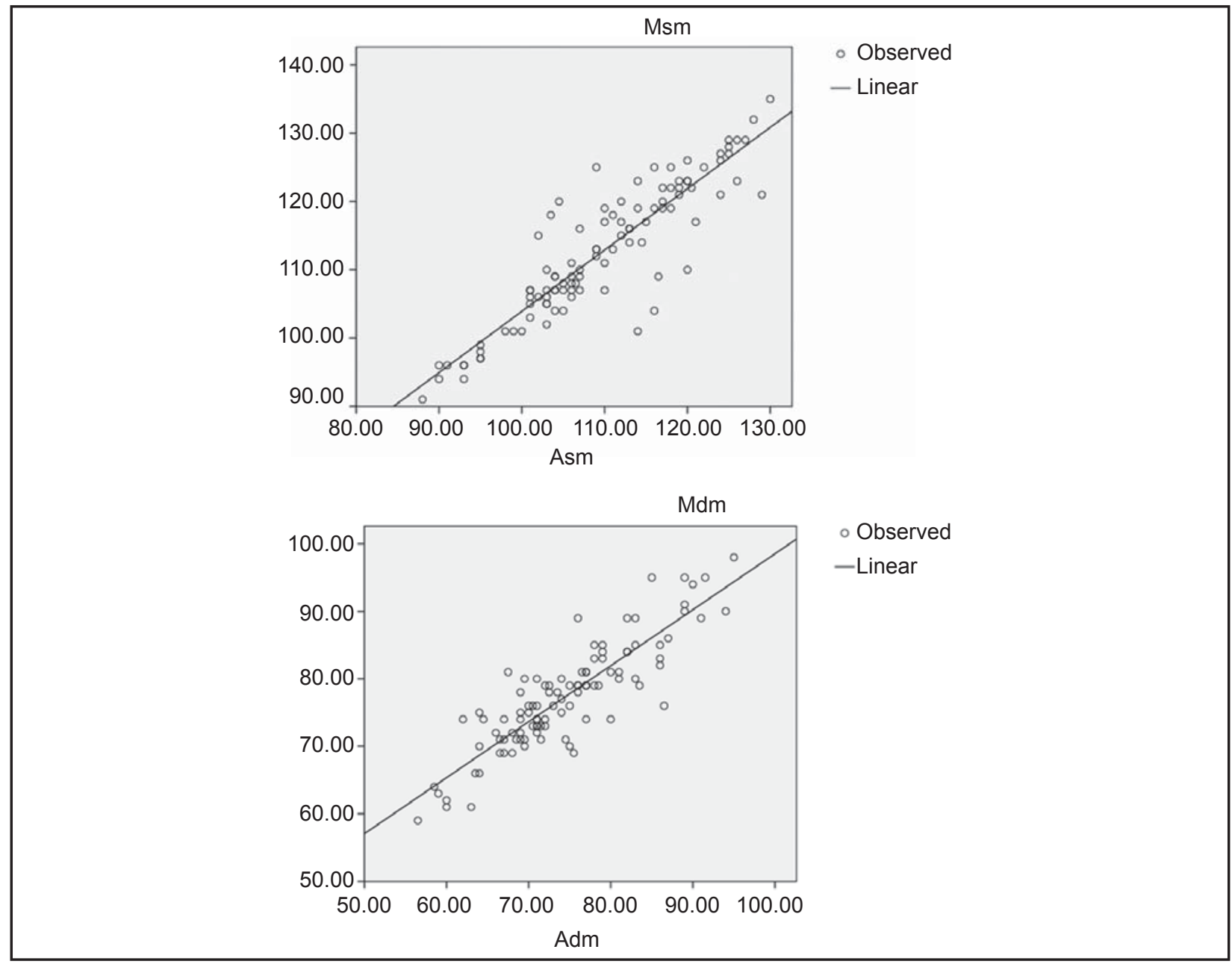

Fig 3: A linear regression analysis was performed to examine the relationship between the automated and manual blood pressure (BP) readings with the automated systolic and diastolic BP as the independent variables.

\section{Discussion}

The prevalence of systemic hypertension in childhood is $1-2 \%$ in the developed countries. Similar data is lacking from India, small surveys in school children suggest a prevalence ranging from 2-5\%. Normative values for BP are based on Mercury sphygmomanometer, which continues to be the preferred method for BP estimation. It is recommended that BP devices be calibrated and validated regularly. The oscillometric devices are increasingly used in infants (in whom auscultation is difficult) and in intensive care settings when frequent BP measurements are needed. However, most oscillometric devices are not validated for children and their normative data based are not available $e^{1,2,3,4}$. In the literature, there is considerable debate in the accuracy of automated BP machines $5,6,7,8$. However, the level of agreement between automated and manual BP measurements can vary for systolic and diastolic readings, and in relation to type of machine and patient population.
The study done by Sigurdsson JA et al $^{9}$ found that the agreement between the automatic BP and the conventional mercury sphygmomanometer in adult is unsatisfactory for clinical purposes and therefore the methods are not interchangeable. Shibata $\mathrm{K}$ et $\mathrm{al}^{10}$ concluded that the reproducibility of BP measured with mercury sphygmomanometer was less than $4 \%$, with each Digital sphygmomanometer less than $9 \%$. In another study, Rotch AL et al ${ }^{11}$ reported that compared with the mercury sphygmomanometer, the automatic monitor was the most accurate in measuring. Menezes $\mathrm{AM}$ et $\mathrm{al}^{12}$ reported the digital device showed a high level of agreement with the mercury manometer when measuring systolic BP. The level of agreement was lower for diastolic BP. In our study, we found high level of agreement between BP measured by manual mercury sphygmomanometer and automated devices. Previous studies ${ }^{13,14,15,16}$ comparing the Omron (automated) device with mercury consistently showed lower readings of Omron (automated) for both systolic and diastolic BP. The Ostchega $\mathrm{Y}$ et $\mathrm{al}^{17}$ reported 
that the Omron (automated) and mercury device measurements were correlated ( $r=0.92$ for systolic BP and $r=0.79$ for diastolic BP), same finding was found in our study also ( $r=0.89$ for systolic BP and $r=0.82$ for diastolic BP).

\section{Conclusion}

This study concludes that the automated blood pressure device is reliable and accurate for measuring the BP in children. Also, we can use this device in the paediatric Out patients department (OPD) and In patients department (IPD) as there is no risk of mercury leakage and also reduces individual differences in measurement methods (human error).

Acknowledgements: None

Funding: None

Conflict of Interest: None

Permission from IRB/Ethical committee: The research was approved by an Institutional Ethics Committee.

\section{References}

1. Lande MB. Systemic Hypertension. In: Kleigman RM, Stanton B, Geme J, Schor N, Behrman R, editors. Nelson Textbook of Pediatrics. $19^{\text {th }} \mathrm{Ed}$. Philadelphia: Elsivier Saunders,2013, p.1639-47.

2. Munter P, He J, Cutler JA, Wildman RP, Whelton BK. Trends in blood pressure among children and adolescents. JAMA 2004;291:2107-113.

3. Jones DW, Appel LJ, Sheps SG, Roccella EJ, Lenfant C. Measuring blood pressure accurately. JAMA 2003;289:1027-30.

4. Mohan B, Kumar N, Aslam N, Rangbulla A, Kumbkarni S, Sood NK, et al. Prevalence of sustained hypertension and obesity in urban and rural school going children in Ludhiana. Indian Heart J 2004;56:310-14.

5. Bagga A, Jain A, Vijayakumar M, Kanitkar M, Ali $U$. Evaluation and Management of Hypertension. Indian Pediatr 2007;103:103-21.

6. Butani L, Morgenstern BZ. Are pitfalls of oscillometric blood pressure measurements preventable in children? Pediatr Nephrol 2003;18:313-18.

7. Carney S. L, Gillies A H, Green S L, Paterson O, Taylor M. S, Smith A J. Hospital blood pressure measurement: staff and device assessment. J Qual Clin Pract 1999;19:95-8.
8. O’Brien E, Waeber B, Parati G, Staessen J, Myers M G. Blood pressure monitoring devices: Recommendations of the European Society of Hypertension. BMJ 2001;322:531-36.

9. Sigurdsson JA, Adalsteinsson B, Hardarson $\mathrm{T}$, Kristinsson A. Blood pressure at the clinic, work and home. Are there white coats at work? Laeknabladid 1996;82(5):371-77.

10. Shibata K, Namikawa K, Murase C. Basic study on blood pressure measurements with a digital sphygmomanometers. Kango Kenkyu 1997;30(4):69-75.

11. Rotch AL, Dean JO, Kendrach MG, Wright SG, Woolley TW. Blood pressure monitoring with home monitors versus mercury sphygmomanometer. Ann Pharmacother 2001;35(7-8):817-22.

12. Menezes AM, Dumith SC, Noal RB, Nunes AP, Mendonça $\mathrm{Fl}$, et al. Validity of a wrist digital monitor for blood pressure measurement in comparison to a mercury sphygmomanometer. Arq Bras Cardiol 2010;94(3):345-9.

13. Coleman A, Freeman $P$, Steel $S$, Shennan A. Validation of the Omron 705IT (HEM-759-E) oscillometric blood pressure monitoring device according to the British Hypertension Society protocol. Blood Press Monit 2006;11(1):27-32.

14. White WB, Anwar YA. Evaluation of the overall efficacy of the Omron office digital blood pressure HEM- 907 monitor in adults. Blood Press Monit 2001;6(2):107-10.

15. Ombani S, Riva I, Giglio A, Caldara G, Groppelli A, Parati G. Validation of the Omron M5-I, R5-I and HEM-907 automated blood pressure monitors in elderly individuals according to the international protocol of the European Society of Hypertension. Blood Press Monit 2007;12(4):233-42.

16. El Assaad MA, Topouchian JA, Darné BM, Asmar RG. Validation of the Omron HEM-907 device for blood pressure measurement. Blood Press Monit 2002;7(4):237-41.

17. Ostchega Y, Guangyu Zhang, Paul Sorlie, Hughes HP, Debra S. Reed-Gillette, Tatiana Nwankwo, Sarah Yoon. Blood Pressure Randomized Methodology Study Comparing Automatic Oscillometric and Mercury Sphygmomanometer Devices: National Health and Nutrition Examination Survey, 20092010. Division of Health and Nutrition Examination Surveys. CDC, Number 59, October 5, 2012, pg.115. [cited 2014 June 20]. (Available from: www. cdc.gov/nchs/data/nhsr/nhsr059.pdf). 